\title{
Fixed point theorems and demiclosedness principle for mappings of asymptotically nonexpansive type in Banach spaces
}

\author{
Caixia Deng, Jingxin Zhang ${ }^{*}$ and Yunan Cui
}

\author{
* Correspondence: zhjx_19@yahoo. \\ com.cn \\ Department of Mathematics, \\ Harbin University of Science and \\ Technology, Harbin 150080, \\ People's Republic of China
}

\begin{abstract}
In this article, we prove the existence of fixed points for the mappings of asymptotically non-expansive type in a Banach space with its characteristic of noncompact convexity associated to the separation measure of noncompactness less than the weakly convergent sequence coefficient. We also verify the demiclosedness principle for mappings of asymptotically nonexpansive type in Banach spaces with locally uniform Opial condition.

Mathematics Subject Classification: 47H09; 47H10.

Keywords: asymptotically nonexpansive type, characteristic of noncompact convexity, weakly convergent sequence coefficient, demiclosedness principle, Opial?'?s condition.
\end{abstract}

\section{Introduction}

In 1974, Kirk [1] introduced the mappings of asymptotically nonexpansive type and proved the existence of fixed points in Banach spaces with the characteristic of convexity less than one. In 1992, You and Xu [2] extended Kirk's theorem to $k$-uniformly rotund ( $k$-UR) Banach spaces for $1<k<\infty$. In 1991, Xu [3] proved the existence of fixed points of the mappings of asymptotically nonexpansive type in nearly uniformly convex (NUC) Banach spaces. In 2002, Li and Sims [4] proved that continuous mappings of asymptotically nonexpansive type has a fixed point in a Banach space with uniform normal structure (see also [5]). In 2004, Zeng [6] proved that if $X$ is uniformly convex in every direction and has weak uniform normal structure then every mapping of asymptotically nonexpansive type has a fixed point.

One of the fundamental and celebrated results in the theory of nonexpansive mappings is Browder's demiclosedness principle [7] which states that if $X$ is a uniformly convex Banach space, $C$ is a nonempty closed convex subset of $X$, and $T: C \rightarrow X$ is a nonexpansive mapping, then $I-T$ is demiclosed at each $y \in X$, that is, for any sequence $\left\{x_{n}\right\}$ in $C$ conditions $x_{n} \stackrel{w}{\rightarrow} x$ and $(I-T) x_{n} \rightarrow y$ imply that $(I-T) x=y$. (Here $I$ is the identity operator of $X$ into itself.) The principle is also valid in a space satisfying Opial's condition. It has been known that the demiclosedness principle plays a key role in studying the asymptotic and ergodic behavior of nonexpansive mapping, see for example [8-10]. In 1991, Xu [3] proved that the demiclosedness principle is

(c) 2012 Deng et al; licensee Springer. This is an Open Access article distributed under the terms of the Creative Commons Attribution License (http://creativecommons.org/licenses/by/2.0), which permits unrestricted use, distribution, and reproduction in any medium, provided the original work is properly cited. 
valid for asymptotically non-expansive mappings in uniformly convex Banach spaces. In 1995, Lin et al. [11] verified the demiclosedness principle for asymptotically nonexpansive mappings in Banach spaces with locally uniform Opial condition.

In this article, we state some fixed point theorems for mappings of asymptotically nonexpansive type, which are more general than the previous results. In Section 3, we give a fixed point theorem for a mapping of asymptotically nonexpansive type in the framework of a reflexive Banach space whose characteristic of noncompact convexity associated to the separation measure of noncompactness is less than the weakly convergent sequence coefficient. If, in addition, $X$ satisfies the nonstrict Opial condition and its characteristic of noncompact convexity associated to the Hausdorff measure of noncompactness less that 1, the conclusion is also valid. In Section 4, we prove that the demiclosedness principle is valid for mappings of asymptotically nonexpansive type in Banach spaces with locally uniform Opial condition. We also show that a NUC Banach space $X$ with Opial's condition satisfies locally uniform Opial condition.

\section{Preliminaries}

Let $X$ be a Banach space $X$. The asymptotically nonexpansive mappings were introduced in 1972 by Geobel and Kirk [12].

Definition 2.1. Let $C$ be bounded subset of $X$. A mapping $T: C \rightarrow C$ is called asymptotically nonexpansive if there exists a sequence $\left\{k_{n}\right\}$ of positive real numbers with $k_{n}$ $\rightarrow 1$ as $n \rightarrow \infty$ for which

$$
\left\|T^{n} x-T^{n} y\right\| \leq k_{n}\|x-y\|, \quad \text { for all } x, y \in C .
$$

If (2.1) is valid for the sequence $\left\{k_{n}\right\}$ with all $k_{n}=1, T$ is said to be nonexpansive.

The mappings of asymptotically nonexpansive type in Banach spaces were defined in 1974 by Kirk [1].

Definition 2.2. Let $C$ be a bounded subset of $X$. A mapping $T: C \rightarrow C$ is called of asymptotically nonexpansive type if $T$ satisfies

$$
\limsup _{n \rightarrow \infty} \sup _{y \in C}\left\{\left\|T^{n} x-T^{n} y\right\|-\|x-y\|\right\} \leq 0
$$

for each $x \in C$, and $T^{N}$ is continuous for some $N \in \mathbb{N}$.

Obviously, asymptotically nonexpansive mappings are mappings of asymptotically nonexpansive type.

The Hausdorff and the separation measures of noncompactness of a nonempty bounded subset $B$ of $X$ are respectively defined as

$$
\begin{aligned}
& \chi(B)=\inf \{d>0: B \text { can be covered by finitely many balls of radius } \leq d\}, \\
& \beta(B)=\sup \left\{\varepsilon>0 \text { : there exists a sequence }\left\{x_{n}\right\} \text { in } B \text { such that sep }\left(\left\{x_{n}\right\}\right) \geq \varepsilon\right\},
\end{aligned}
$$

where

$$
\operatorname{sep}\left(\left\{x_{n}\right\}\right)=\inf \left\{\left\|x_{n}-x_{m}\right\|: n \neq m\right\} .
$$

The modulus of noncompact convexity associated to $\varphi(\varphi=\chi$, or $\beta)$ is defined in the following way: 


$$
\Delta_{X, \phi}(\varepsilon)=\inf \left\{1-d(0, A): A \subset B_{X} \text { is convex, } \phi(A) \geq \varepsilon\right\} .
$$

The characteristic of noncompact convexity of $X$ associated with the measure of non-compactness $\varphi$ is defined by

$$
\varepsilon_{\phi}(X)=\sup \left\{\varepsilon \geq 0: \Delta_{X, \phi}(\varepsilon)=0\right\} .
$$

When $X$ is a reflexive Banach space we have the following alternative expression for the modulus of noncompact convexity associated with $\chi$ and $\beta$,

$$
\begin{aligned}
& \Delta_{X, \chi}(\varepsilon)=\inf \left\{1-\|x\|:\left\{x_{n}\right\} \subset B_{X}, x=w-\lim _{n} x_{n}, \chi\left(\left\{x_{n}\right\}\right) \geq \varepsilon\right\}, \\
& \Delta_{X, \beta}(\varepsilon)=\inf \left\{1-\|x\|:\left\{x_{n}\right\} \subset B_{X}, x=w-\lim _{n} x_{n}, \operatorname{sep}\left(\left\{x_{n}\right\}\right) \geq \varepsilon\right\} .
\end{aligned}
$$

It is known that $X$ is $N U C$ if and only if $\varepsilon_{\varphi}(X)=0$, where $\varphi$ is $\beta$ or $\chi$. Another important fact is that if $\varepsilon_{\beta}(X)<1$, then $X$ is reflexive. The above-mentioned definitions and properties can be found in [13].

Recall that a Banach space $X$ is said to satisfy Opial condition [14] if for each sequence $\left\{x_{n}\right\}$ in $X$ the condition $x_{n} \stackrel{w}{\rightarrow} x$ implies that

$$
\limsup _{n \rightarrow \infty}\left\|x_{n}-x\right\|<\limsup _{n \rightarrow \infty}\left\|x_{n}-y\right\|
$$

for all $y \neq x$. If $<$ is replaced by $\leq$ in the above inequality, $X$ is said to satisfy nonstrict Opial condition. In 1992, Prus [15] introduced uniform Opial condition. A Banach space $X$ is said to satisfy the uniform Opial condition if for each $c>0$, there exists an $r$ $>0$ such that

$$
1+r \leq \liminf _{n \rightarrow \infty}\left\|x_{n}+x\right\|
$$

for each $x \in X$ with $\|x\| \geq c$ and each sequence $\left\{x_{n}\right\}$ in $X$ such that $w-\lim _{n \rightarrow \infty} x_{n}=0$ and $\lim _{\inf _{n \rightarrow \infty}}|| x_{n} \| \geq 1$.

In 1995, Lin, Tan and Xu [11] first introduced the locally uniform Opial condition. In 2003, Fetter and Gamboa de Buen [16] amended the locally uniform Opial condition: a Banach space $X$ is said to satisfy the locally uniform Opial condition if for any weakly null sequence $\left\{x_{n}\right\}$ in $X$ with $\lim \sup _{n \rightarrow \infty}|| x_{n} \| \geq 1$ and any $c>0$, there is $r>0$ such that

$$
1+r \leq \limsup _{n \rightarrow \infty}\left\|x_{n}+x\right\|
$$

for every $x \in X$ with $\|x\| \geq c$. Clearly, uniform Opial condition implies local uniform Opial condition, which in turn implies Opial's condition. The following equivalent definition for local uniform Opial condition holds.

Proposition 2.3. [[16], Theorem 1.3] A Banach space $X$ is said to satisfy the locally uniform Opial condition if and only if for any sequence $\left\{x_{n}\right\}$ in $X$ which converges weakly to $x \in X$ and for any sequence $\left\{y_{m}\right\}$ in $X$, the condition

$$
\limsup _{m \rightarrow \infty} \limsup _{n \rightarrow \infty}\left\|x_{n}-y_{m}\right\| \leq \limsup _{n \rightarrow \infty}\left\|x_{n}-x\right\|
$$

implies that $\left\{y_{m}\right\}$ converges to $x$ in norm. 
We also recall the Bynum's weakly convergent sequence coefficient. The asymptotic diameter and asymptotic radius of a sequence $\left\{x_{n}\right\}$ in a Banach space $X$ are defined by

$$
\begin{aligned}
& \operatorname{diam}_{a}\left(\left\{x_{n}\right\}\right)=\lim _{k \rightarrow \infty}\left\{\sup \left\|x_{n}-x_{m}\right\|: n, m \geq k\right\}, \\
& r_{a}\left(\left\{x_{n}\right\}\right)=\inf \left\{\limsup _{n \rightarrow \infty}\left\|x_{n}-y\right\|: y \in \overline{\operatorname{conv}}\left(\left\{x_{n}\right\}\right)\right\} .
\end{aligned}
$$

The weakly convergent sequence coefficient [17] of $X$ is defined by

$$
\operatorname{WCS}(X)=\inf \left\{\frac{\operatorname{diam}_{a}\left(\left\{x_{n}\right\}\right)}{r_{a}\left(\left\{x_{n}\right\}\right)}\right\}
$$

where the infimum is taken over all sequences $\left\{x_{n}\right\}$ weakly convergent to zero which are not norm convergent to zero. It is clear that $1 \leq \operatorname{WCS}(X) \leq 2$. The definition of WCS $(X)$ above does not make sense if the space $X$ has the Schur property but in that case we may say by convention that $\operatorname{WCS}(X)=2$. In this article, we use the following equivalent formulation (see [18]

$$
\operatorname{WCS}(X)=\inf \left\{\lim _{n, m ; n \neq m}\left\|x_{n}-x_{m}\right\|\right\}
$$

where the infimum is taken over all weakly null sequences $\left\{x_{n}\right\} \subset X$ with $\left\|x_{n}\right\| \rightarrow 1$ and $\lim _{n, m ; n \neq m}|| x_{n}-x_{m} \|$ exists.

\section{Fixed points and moduli of noncompact convexity}

Let $C$ be a nonempty weakly compact convex subset of a Banach space $X$, and $T: C \rightarrow$ $C$ be a mapping of asymptotically nonexpansive type. Denote by $\mathfrak{F}$ the family of all closed convex nonempty subsets $K$ of $C$ with the following property $(\omega)$ :

$$
x \in K \Rightarrow \omega_{w}(x) \subset K,
$$

where

$$
\omega_{w}(x):=\left\{y \in X: y=w-\lim _{i \rightarrow \infty} T^{n_{i}} x \text { for some } n_{i} \uparrow \infty\right\}
$$

is the weak $\omega$-limit set of $T$ at $x$. Let $\mathfrak{F}$ be ordered by inclusion. The weak compactness of $C$ allows us to use Zorn's Lemma to obtain a minimal element (say) $K$ in $\mathfrak{F}$. To prove the theorem we use the following lemma from [3] (see also [[4], Lemma 1]).

Lemma 3.1. Let $C$ be a nonempty subset of a Banach space $X$ and let $T$ be a mapping of asymptotically nonexpansive type $C$. Suppose there exists a nonempty bounded closed convex subset $E$ of $C$ with the property $(\omega)$. Then there is a closed convex nonempty subset $K$ of $C$ and $a r>0$ such that:

(i) if $x \in K$; then every weak limit point of $\left\{T^{n} x\right\}$ is contained in $K$;

(ii) $r_{x}(y)=r$ for all $x, y \in K$; where $r_{x}$ is the functional defined by

$$
r_{x}(y)=\limsup _{n \rightarrow \infty}\left\|T^{n} x-y\right\|, y \in X .
$$


Remark 3.2. From [3], the set $K$ in Lemma 3.1 is the above minimal element obtained by Zorn' Lemma. In fact, $r$ and $K$ are respectively the asymptotic radius and asymptotic center of the sequence $\left\{T^{n} x\right\}$ with respect to $C$.

From Kirk's proof of Theorem 2 in [1], we have the following lemma.

Lemma 3.3. (Kirk [1]) Let $C$ be a nonempty weakly compact convex subset of a Banach space $X$, and $T: C \rightarrow C$ be a mapping of asymptotically nonexpansive type and $T^{N}$ be continuous for some $N \in \mathbb{N}$. If there exists a nonempty compact convex subset $K$ of $C$ satisfying that

$$
x \in K \Rightarrow \varnothing \neq \omega_{\|\cdot\|}(x) \subset K,
$$

where

$$
\omega_{\|\cdot\|}(x)=\left\{y \in X: y=\|\cdot\|-\lim _{i \rightarrow \infty} T^{n_{i}} x \text { for some } n_{i} \uparrow \infty\right\},
$$

then $T$ has a fixed point in $C$.

Theorem 3.4. Let $C$ be a nonempty bounded closed convex subset of a reflexive Banach space $X$ and $T: C \rightarrow C$ be a mapping of asymptotically nonexpansive type and $T^{N}$ be continuous for some $N \in \mathbb{N}$. If $\varepsilon_{\beta}(X)<\mathrm{WCS}(X)$, then $T$ has a fixed point in $C$.

Proof. Since $X$ is reflexive, $C$ is a nonempty weakly compact convex subset of $X$. Denote by $\mathfrak{F}$ the family of all closed convex nonempty subsets $K$ of $C$ with the property $(\omega)$ :

$$
x \in K \Rightarrow \omega_{w}(x) \subset K,
$$

where $\omega_{w}(x)$ is the weak $\omega$-limit set of $T$ at $x$. By the weakly compactness of $C$, we have $C \in \mathfrak{F}$, so $\mathfrak{F} \neq \emptyset$. Let $\mathfrak{F}$ be ordered by inclusion. Using Zorn's Lemma, we obtain a minimal element $K$ in $\mathfrak{F}$. Then $K$ be a minimal subset of $C$ with respect to being nonempty, closed, convex and satisfying the property $(\omega)$.

Since $x \in K$ implies $\omega_{w}(x) \subset K$, thus $\omega_{\|} \cdot \|(x) \subset K$. By Lemma 3.3, we only need to show that for each $x \in K,\left\{T^{n} x\right\}$ admits a norm-convergence subsequence, thus $\omega_{\|\cdot\|}(x) \neq 0$.

If $r=0$, it is easy to see that $\left\{T^{n} x\right\}$ converges in norm to a fixed point of $T$ for any $x$ $\in K$. Now we assume that $r>0$. Let $x$ be arbitrary element in $K$ and $\left\{n_{i}\right\}$ be a subsequence of the positive integers $\{n\}$. If $\left\{T^{n_{i}} x\right\}$ contains no norm-convergent subsequence, then we may assume without loss of generality that it has a subsequence, denoted by $\left\{x_{n}\right\}$, such that $\lim _{n \neq m}\left\|x_{n}-x_{m}\right\|$ exists Since $X$ is reflexive, we may assume that $\left\{x_{n}\right\}$ converges weakly to some $z \in K$. Thus, by Lemma 3.1 and the weak lower semicontinuity of the norm, we have

$$
r=\limsup _{n \rightarrow \infty}\left\|x_{n}-z\right\| \leq \limsup _{n \rightarrow \infty} \liminf _{m \rightarrow \infty}\left\|x_{n}-x_{m}\right\|=\lim _{n \neq m}\left\|x_{n}-x_{m}\right\| .
$$

Since lim $\sup _{n \rightarrow \infty}|| T^{n} x-x\left\|=\lim \sup _{n \rightarrow \infty}\right\| T^{n} x-z \|=r$, for any $\eta>0$, we can find an $n_{0}$ so large that for $n \geq n_{0}$

$$
\left\|T^{n} x-x\right\|<r+\frac{1}{2} \eta,\left\|T^{n} x-z\right\| \geq r-\eta
$$


and

$$
\sup \left\{T^{n} x-T^{n} u\|-\| x-u \|: u \in C\right\}<\frac{1}{2} \eta
$$

We write $x_{k}=T^{n(k)} x, k \geq 1$. For an arbitrary but fixed $n \geq n_{0}$, choose a $k_{0}$ large enough so that $m(k) \geq n+n_{0}$ for $k \geq k_{0}$. Now it follows that for $k \geq k_{0}$,

$$
\begin{aligned}
\left\|T^{n} x-x_{k}\right\| & =\left\|T^{n} x-T^{n}\left(T^{m(k)-n} x\right)\right\| \\
& <\frac{1}{2} \eta+\left\|x-T^{m(k)-n} x\right\| \\
& <r+\eta .
\end{aligned}
$$

Since $\lim _{n \neq m}|| x_{n}-x_{m}||=\mathrm{d} \geq r>0$, we suppose without loss of generality that $\| x_{k}$ $x_{l} \| \geq d-\eta$ for all $k \neq l$.

Now we define a sequence $\left\{w_{k}\right\}$ by $w_{k}=\left(T^{n} x-T^{m(k)} x\right) /(r+\eta)$. Then we know that \| $w_{k} \| \leq 1$ for $k \geq k_{0},\left\{w_{k}\right\}$ converges weakly to $\left(T^{n} x-z\right) /(r+\eta)$, and sep $\left(\left\{w_{k}\right\}\right)=$ sep $\left(\left\{\left(T^{m(k)} x\right) /(r+\eta)\right\}\right) \geq(d-\eta) /(r+\eta)$. Thus we conclude from the definition of $\Delta_{X, \beta}(\cdot)$ that

$$
\Delta_{X, \beta}\left(\frac{d-\eta}{r+\eta}\right) \leq 1-\left\|\frac{T^{n} x-z}{r+\eta}\right\| \leq 1-\frac{r-\eta}{r+\eta}
$$

for all $n \geq n_{0}$. Since the last inequality is true for any $\eta>0$, we obtain $\Delta_{X, \beta}(d / r)=0$, whence $\varepsilon_{\beta}(X) \geq d / r$. Now we estimate $d$ as follows:

$$
\begin{aligned}
d & =\lim _{k \neq l}\left\|x_{k}-x_{l}\right\|=\lim _{k \neq l}\left\|\left(x_{k}-z\right)-\left(x_{l}-z\right)\right\| \\
& \geq \operatorname{WCS}(X) \limsup _{n \rightarrow \infty}\left\|x_{n}-z\right\| \\
& \geq \operatorname{WCS}(X) \cdot r .
\end{aligned}
$$

Hence, $\varepsilon_{\beta}(X) \geq \operatorname{WCS}(X)$. This is a contradiction.

The following Lemma can be found in the proof of Theorem 3.4 in [19].

Lemma 3.5. Let $X$ be a Banach space with nonstrict Opial condition, and let $\left\{x_{n}\right\}$ be a sequence weakly convergent to a point $z$. Then

$$
\chi\left(\left\{x_{n}\right\}\right)=\limsup _{x \rightarrow \infty}\left\|x_{n}-z\right\| .
$$

Theorem 3.6. Let $C$ be a nonempty bounded closed convex subset of a reflexive Banach space $X$ and $T: C \rightarrow C$ be a mapping of asymptotically nonexpansive type and $T^{N}$ be continuous for some $N \in \mathbb{N}$. If $X$ satisfies the nonstrict Opial condition and $\varepsilon_{\chi}$ $(X)<1$, then $T$ has a fixed point.

Proof. As in the proof of the Theorem 3.4, if $X$ satisfies the nonstrict Opial condition, by Lemma 3.5, we also have

$$
\chi\left(\left\{\omega_{k}\right\}\right)=\chi\left(\left\{\frac{T^{m(k)} x}{r+\eta}\right\}\right)=\limsup _{k \rightarrow \infty}\left\|\frac{T^{m(k)} x-z}{r+\eta}\right\| \geq \frac{r-\eta}{r+\eta} .
$$

Therefore, the conclusion holds. 


\section{Demiclosedness principle for mappings of asymptotically nonexpansive type}

In this section we prove the demicloseness principle for mappings of asymptotically nonexpansive type either in a Banach space with locally uniform Opial condition or in a NUC Banach space with Opial's condition.

Lemma 4.1. Suppose $X$ is a Banach space satisfying Opial's conditon and $C$ is a nonempty weakly compact convex subset of $X$, and $T: C \rightarrow C$ is a continuous mapping of asymptotically nonexpansive type. Suppose also that $\left\{x_{n}\right\}$ is a sequence in $C$ which converges weakly to $x$ and for which the sequence $\left\{x_{n}-T x_{n}\right\}$ converges strongly to zero. Then $\left\{T^{n} x\right\}$ converges weakly to $x$.

Proof. For each integer $m \geq 1$, set

$$
A_{m}=\overline{\mathrm{CO}}\left\{T^{i} x: i \geq m\right\} \quad \text { and } \quad A=\bigcap_{m=1}^{\infty} A_{m} .
$$

Since $C$ is weakly compact, $A$ is nonempty and it is readily seen that $A=\overline{\mathrm{CO}} \omega_{w}(x)$, where $\omega_{w}(x)$ is the weak $\omega$-limit set of $\mathrm{T}$ at $x$, i.e., the set

$$
\left\{y \in X: y=w-\lim _{j \rightarrow \infty} T^{n_{j}} x \text { for some } n_{j} \uparrow \infty\right\} .
$$

Showing the weak convergence of $\left\{T^{n} x\right\}$ to $x$ is equivalent to showing that $A=\{x\}$. To this end, let the functional $f$ be defined by

$$
f(y)=\limsup _{n \rightarrow \infty}\left\|x_{n}-y\right\|, y \in X .
$$

If there exists $y_{0} \in A$ such that $y_{0} \neq x$, then by Opial's condition, $f\left(y_{0}\right)>f(x)$. Let $\eta:=$ $f\left(y_{0}\right)-f(x)>0$. Noticing that $T$ is a mapping of asymptotically nonexpansive type, we denote

$$
c_{m}=\max \left\{0, \sup _{x, y \in C}\left(\left\|T^{m} x-T^{m} y\right\|-\|x-y\|\right)\right\} .
$$

Then $\lim _{m \rightarrow \infty} c_{m}=0$. Thus there exists $m_{0} \in \mathbb{N}$ such that $c_{m}<\eta / 2$ for all $m \geq m_{0}$. Since $y_{0} \in A_{m_{0}+1}$, there exist an integer $p \geq 1$ and nonnegative numbers $t_{1}, \ldots, t_{p}$ with $\sum_{i=1}^{p} t_{i}=1$ such that

$$
\left\|y_{0}-\sum_{j=1}^{p} t_{i} T^{m_{0}+j} x\right\|<\frac{\eta}{2}
$$


It follows that

$$
\begin{aligned}
f\left(y_{0}\right) & =\limsup _{n \rightarrow \infty}\left\|x_{n}-y_{0}\right\| \\
& \leq \limsup _{x \rightarrow \infty}\left\|y_{0}-\sum_{j=1}^{p} t_{j} T^{m_{0}+j} x\right\|+\underset{n \rightarrow \infty}{\limsup }\left\|x_{n}-\sum_{j=1}^{p} t_{j} T^{m_{0}+j} x\right\| \\
& <\frac{\eta}{2}+\sum_{j=1}^{p} t_{j} \limsup _{n \rightarrow \infty}\left\|x_{n}-T^{m_{0}+j} x\right\| \\
& =\frac{\eta}{2}+\limsup _{n \rightarrow \infty}\left\|T^{m_{0}+j} x_{n}-T^{m_{0}+j} x\right\| \\
& \leq \frac{\eta}{2}+c_{m_{0}+j}+\underset{n \rightarrow \infty}{\limsup _{n \rightarrow \infty}\left\|x_{n}-x\right\|} \\
& <\eta+f(x)=f\left(y_{0}\right) .
\end{aligned}
$$

This contradiction shows that we must have $A=\{x\}$.

Theorem 4.2. Suppose $X$ is a Banach space satisfying the locally uniform Opial condition, $C$ is a nonempty weakly compact convex subset of $X$, and $T: C \rightarrow C$ is a mapping of asymptotically nonexpansive type and $T^{N}$ is continuous for some $N \in \mathbb{N}$. Then $I$ - $T$ is demiclosed at zero, i.e., if $\left\{x_{n}\right\}$ is a sequence in $C$ which converges weakly to $x$ and if the sequence $\left\{x_{n}-T x_{n}\right\}$ converges strongly to zero, then $x-T x=0$.

Proof. Let $F=T^{N}$, then $F$ is a continuous mapping of asymptotically nonexpansive type. Suppose that $\left\{x_{n}\right\}$ is a sequence in $C$ such that $x_{n} \stackrel{w}{\rightarrow} x$ and $x_{n}-T x_{n} \rightarrow 0$, then $x_{n}-F x_{n} \rightarrow 0$. By Lemma 4.1 , we have $F^{n} x \stackrel{w}{\rightarrow} x$. Since $F$ is a mapping of asymptotically nonexpansive type, it follows that

$$
\begin{aligned}
& \limsup _{m \rightarrow \infty}\left(\limsup _{n \rightarrow \infty}\left\|F^{n} x-F^{m} x\right\|\right) \\
= & \limsup _{m \rightarrow \infty}\left(\limsup _{n \rightarrow \infty}\left\{\left(\left\|F^{m} x-F^{m}\left(F^{n-m} x\right)\right\|-\left\|x-F^{n-m} x\right\|\right)+\left\|F^{n-m} x-x\right\|\right\}\right) \\
\leq & \limsup _{m \rightarrow \infty}\left(\sup _{u \in C}\left\{\left\|F^{m} x-F^{m} u\right\|-\|x-u\|\right\}\right)+\limsup _{m \rightarrow \infty}\left\|F^{n} x-x\right\| \\
= & \limsup _{m \rightarrow \infty}\left\|F^{n} x-x\right\| .
\end{aligned}
$$

Observing that $X$ satisfies the locally uniform Opial condition, by Proposition 2.3, we have $F^{n} x \rightarrow x$ and, hence, $F x=x$ by continuity of $F$. Thus we have shown that $x$ is a fixed point of $T^{N}$.

Next, we will prove that $x$ is also a fixed point of $T$. Let $K$ be the minimal subset of $C$ with respect to being nonempty, closed, convex and satisfying the property $(\omega)$. From the proof of Lemma 4.1 , we have $x \in K$. Let $D=\left\{x, T x, \ldots, T^{N-1} x\right\}$. It is clear that $D$ is contained in $K$. If $\operatorname{diam}(D)>0$, as compact convex sets have normal structure, there exists $u \in \overline{\mathrm{CO}} D \subset K$ such that

$$
r:=\sup _{\gamma \in D}\|u-\gamma\|<\operatorname{diam}(D) .
$$

Let

$$
E=\left\{z \in K: \sup _{\gamma \in D}\|z-y\| \leq d\right\} .
$$


Then $E$ is nonempty $(u \in E)$, closed and convex subset of $K$. Also $E$ is a proper subset of $K$ because of (4.1). Now we claim that $E$ has property $(\omega)$. Indeed, if we take any $x \in E$ and $v \in \omega_{w}(x)$, i.e., there exists a subsequence $\left\{n_{i}\right\}$ such that $T^{n_{i}} \stackrel{w}{\rightarrow} v$ as $n_{i} \rightarrow$ $\infty$, we note that $T(D)=D$. Therefore, it is easily to see that $T^{n}(D)=D$ for all $n \geq 1$. Hence, for any $y \in D$ and $n \geq 1$, there exists $y_{n} \in D$ such that $T^{n} y_{n}=y$. Clearly, it follows that $T^{n_{i}} x-T^{n_{i}} y_{n_{i}}=T^{n_{i}} x-y \stackrel{w}{\rightarrow} v-y$ as $n_{i} \rightarrow \infty$. By the weak lower semicontinuity of the norm, we have

$$
\begin{aligned}
\|v-y\| & \leq \liminf _{i \rightarrow \infty}\left\|T^{n_{i}} x-T^{n_{i}} y_{n_{i}}\right\| \leq \limsup _{n \rightarrow \infty}\left\|T^{n} x-T^{n} y_{n}\right\| \\
& \leq \limsup _{n \rightarrow \infty}\left(\left\|T^{n} x-T^{n} y_{n}\right\|-\left\|x-y_{n}\right\|\right)+\limsup _{n \rightarrow \infty}\left\|x-y_{n}\right\| \\
& \leq \limsup _{n \rightarrow \infty}\left(\sup _{u \in C}\left\{\left\|T^{n} x-T^{n} u\right\|-\|x-u\|\right\}\right)+\sup _{y \in D}\|x-y\| \\
& \leq d,
\end{aligned}
$$

which means that $v \in E$ and hence $\omega_{w}(x) \subset E$. Therefore, $E$ has property $(\omega)$. This then contradicts the minimality of $K$. According to this contradiction, we conclude that $\operatorname{diam}(D)=0$. Thus $x$ is a fixed point of $T$.

Theorem 4.3. Suppose $X$ is a Banach space satisfying the locally uniform Opial condition, $C$ is a nonempty weakly compact convex subset of $X$, and $T: C \rightarrow C$ is a mapping of asymptotically nonexpansive type and $T^{N}$ is continuous for some $N \in \mathbb{N}$. If $T$ is asymptotically regular at some $x \in C$, i.e., $\lim _{n \rightarrow \infty}\left\|T^{n} x-T^{n+1} x\right\|=0$ then $\left\{T^{n} x\right\}$ converges weakly to a fixed point of $T$.

Proof. By Theorem 4.2, the asymptotic regularity of $T$ at $x$ implies that $\omega_{w}(x) \subset$ Fix (T), the fixed point set of $T$. So we only need to show that $\omega_{w}(x)$ is a singleton. Assume that $p=w-\lim _{i \rightarrow \infty} T^{n_{i}} x, q=w-\lim _{j \rightarrow \infty} T^{m_{j}} x$ and $p \neq q$. Since by a routine argument, the limit $\lim _{n \rightarrow \infty}\left\|T^{n} x-y\right\|$ exists for every $y \in \operatorname{Fix}(T)$, by Opial's condition, we have

$$
\begin{aligned}
\lim _{n \rightarrow \infty}\left\|T^{n} x-p\right\| & =\lim _{i \rightarrow \infty}\left\|T^{n_{i}} x-p\right\| \\
& <\lim _{i \rightarrow \infty}\left\|T^{n_{i}} x-q\right\|=\lim _{j \rightarrow \infty}\left\|T^{m_{j}} x-q\right\| \\
& <\lim _{j \rightarrow \infty}\left\|T^{m_{j}} x-p\right\|=\lim _{n \rightarrow \infty}\left\|T^{n} x-p\right\| .
\end{aligned}
$$

This is a contradiction. $\square$

In [11], the authors proved that a $k$-UR Banach space with Opial's condition satisfies the locally uniform Opial condition. In the sequel, we extend this result to NUC Banach spaces.

Lemma 4.4. Suppose $X$ is a NUC Banach space, $C$ is a nonempty closed convex subset of $X,\left\{x_{n}\right\}$ is a bounded sequence in $X$, and $f$ is the functional on $X$ defined by

$$
f(\gamma)=\limsup _{n \rightarrow \infty}\left\|x_{n}-y\right\|, y \in X .
$$

Then every sequence in $C$ which minimizes $f$ over $C$ admits a norm-convergent subsequence.

Proof. Let $I=\inf \{f(y): y \in C\}$ and $\left\{y_{n}\right\}$ be a sequence in $C$ which minimizes $f$ over $C$, i. e., $\lim _{m \rightarrow \infty}\left(y_{m}\right)=I$. We may assume that $I>0$ because otherwise, it is easily seen that 
the whole sequence $\left\{y_{m}\right\}$ is norm-convergent. Now suppose on the contrary that $\left\{y_{m}\right\}$ does not contain any norm-convergent subsequence. Then there exist $\rho>0$ and a subsequence $\left\{z_{m}\right\}$ such that

$$
\operatorname{sep}\left(\left\{z_{m}\right\}\right)=\inf \left\{\left\|z_{n}-z_{m}\right\|: n \neq m\right\}=\rho>0 .
$$

Since $X$ is reflexive, we may assume that $\left\{z_{m}\right\}$ converges weakly to some $z$ in $C$. Noticing that $X$ is NUC, we can choose $\varepsilon$ small enough such that

$$
(I+\varepsilon)\left(1-\Delta_{X, \beta}\left(\frac{\rho}{I+\varepsilon}\right)\right)<I .
$$

Since $\lim _{m \rightarrow \infty}\left(y_{m}\right)=I$, we can find $m_{0} \in \mathbb{N}$ such that

$$
f\left(y_{m}\right)<I+\varepsilon, \forall m \geq m_{0} .
$$

For any $k \geq 1$, choosing $\left\{z_{m_{k}}\right\}$ such that $m_{k}>m_{0}$, then there exists an integer $n_{0}$ so large that

$$
\left\|x_{n}-z_{m_{k}}\right\|<I+\varepsilon
$$

for all $n \geq n_{0}$ and $k \geq 1$. Define a sequence $\left\{w_{k}\right\}$ by $w_{k}=\left(x_{n}-z_{m_{k}}\right) /(I+\varepsilon)$. Then we know that $\left\|w_{k}\right\| \leq 1$ for $k \geq 1,\left\{w_{k}\right\}$ converges weakly to $\left(x_{n}-z\right) /(I+\varepsilon)$, and $\operatorname{sep}\left(\left\{w_{k}\right\}\right)$ $=\operatorname{sep}\left(\left\{z_{m k} /(I+\varepsilon)\right\}\right) \geq \rho /(I+\varepsilon)$. Thus we conclude that from the definition of $\Delta_{\mathrm{X}, \beta}(\cdot)$ that

$$
\left\|x_{n}-z\right\| \leq(I+\varepsilon)\left(1-\Delta_{X, \beta}\left(\frac{\rho}{I+\varepsilon}\right)\right)<I
$$

for all $n \geq n_{0}$. Hence we have $f(z)<I$. This contradiction shows that the lemma is true.

By Lemma 4.4 and Theorem 3.5 in [11], we have the following theorem.

Theorem 4.5. If $X$ is NUC and satisfies Opial's condition, then it satisfies the locally uniform Opial condition.

By Theorems 4.2 and 4.5, we obtain the following corollary.

Corollary 4.6. Suppose $X$ is a NUC Banach space with Opial's condition, $C$ is a nonempty weakly compact convex subset of $X$, and $T: C \rightarrow C$ is a mapping of asymptotically nonexpansive type and $T^{N}$ is continuous for some $N \geq 1$. Then $I-T$ is demiclosed at zero.

\section{Acknowledgements}

The authors are extremely grateful to the reviewers for careful reading, valuable comment and suggestions that improved the content of this article. This work is supported by the Postdoctoral Foundation of Heilongjiang Province No. LBHZ08102 and the Natural Science Foundation of Heilongjiang Province No. A200902.

\section{Authors' contributions}

YC contributed the ideas and gave some valuable suggestions. JZ participated in the sequence alignment and drafted the manuscript. CD have contributed in improving the main results presented in this article. All authors read and approved the final manuscript.

\section{Competing interests}

The authors declare that they have no competing interests. 


\section{References}

1. Kirk, WA: Fixed point theorems for non-Lipschitzian mappings of asymptotically nonexpansive types. Isr J Math. 17 339-346 (1974). doi:10.1007/BF02757136

2. You, ZY, Xu, HK: K-uniform rotundity and fixed points for mappings of asymptotically nonexpansive type. Chin J Eng Math (in Chinese). 9(4):1-8 (1992)

3. $\mathrm{Xu}, \mathrm{HK}$ : Existence and convergence for fixed points of mappings of asymptotically nonexpansive type. Nonlinear Anal. 16(12):1139-1146 (1991). doi:10.1016/0362-546X(91)90201-B

4. Li, G, Sims, B: Fixed point theorems for mappings of asymptotically nonexpansive type. Nonlinear Anal. 50, 1085-1091 (2002). doi:10.1016/S0362-546X(01)00744-1

5. Kim, T: Fixed point theorems for non-Lipschitzian self-mappings and geometric properties of Banach spaces. Fixed Point Theory Appl. 3, 125-135 (2002)

6. Zeng, L: On the existence of fixed points for mappings of asymptotically nonexpansive type. J Syst Sci Complex. 17(2):188-196 (2004)

7. Browder, FE: Semicontractive and semiaccretive nonlinear mappings in Banach spaces Bull. Am Math Soc. 74, 660-665 (1968). doi:10.1090/S0002-9904-1968-11983-4

8. Reich, S: Weak convergence theorems for nonexpansive mappings in Banach spaces. J Math Anal Appl. 67, 274-276 (1979). doi:10.1016/0022-247X(79)90024-6

9. Hirano, N: A proof of the mean ergodic theorem for nonexpansive mappings in Banach spaces. Proc Am Math Soc. 78 361-365 (1980). doi:10.1090/S0002-9939-1980-0553377-8

10. García-Falset, J, Sims, B, Smyth, MA: The demiclosedness principle for mappings of asymptotically nonexpansive type. Houst J Math. 158, 101-108 (1996)

11. Lin, PK, Tan, KK, Xu, HK: Demiclosedness principle and asymptotic behavior for asymptotically nonexpansive mappings. Nonlinear Anal. 24(6):929-946 (1995). doi:10.1016/0362-546X(94)00128-5

12. Geobel, K, Kirk, WA: A fixed point theorem for asymptotically nonexpansive mappings. Proc Am Math Soc. 35, 171-174 (1972). doi:10.1090/S0002-9939-1972-0298500-3

13. Ayerbe, JM, Domínguez-Benavides, T, López-Acedo, G: Measures of Noncompactness in Metric Fixed Point Theory Birkhäuser-Verlag, Berlin (1997)

14. Opial, Z: Weak convergence of sequence of sesuccessive approximations for nonexpansive mappings. Bull Am Math Soc. 73, 591-597 (1967). doi:10.1090/S0002-9904-1967-11761-0

15. Prus, S: Banach spaces with the uniform Opial property. Nonlinear Anal. 18(8):697-704 (1992). doi:10.1016/0362-546X(92) 90165-B

16. Fetter, H, Gamboa de Buen, B: Locally uniform Opial conditions. Nonlinear Anal. 53, 743-750 (2003). doi:10.1016/S0362546X(03)00023-3

17. Bynum, WL: Normal structure coefficients for Banach spaces. Pac J Math. 86, 427-435 (1980)

18. Sims, B, Smyth, MA: On some Banach space properties sufficient for weak normal structure and their permanence properties. Trans Am Math Soc. 351, 497-513 (1999). doi:10.1090/S0002-9947-99-01862-0

19. Domínguez-Benavides, T, Ramírez, PL: Fixed-point theorems for multivalued non-expansive mappings without uniform convexity. Abstr Appl Anal. 2003(6):375-386 (2003). doi:10.1155/S1085337503203080

doi:10.1186/1687-1812-2012-37

Cite this article as: Deng et al: Fixed point theorems and demiclosedness principle for mappings of

asymptotically nonexpansive type in Banach spaces. Fixed Point Theory and Applications 2012 2012:37.

\section{Submit your manuscript to a SpringerOpen ${ }^{\circ}$ journal and benefit from:}

Convenient online submission

Rigorous peer review

- Immediate publication on acceptance

- Open access: articles freely available online

- High visibility within the field

- Retaining the copyright to your article

Submit your next manuscript at $>$ springeropen.com 